\title{
Evaluation of the Performance of Space Reduction Technique Using AC and DC Models in Transmission Expansion Problems
}

\author{
Phillipe Vilaça Gomes and João Tomé Saraiva \\ INESC TEC and FEUP/DEEC \\ Dept. Eng. Eletrotécnica e de Computadores da Faculdade de Engenharia da Universidade do Porto - Portugal \\ phillipe.gomes@fe.up.pt, jsaraiva@fe.up.pt
}

\begin{abstract}
Transmission Expansion Planning (TEP) is an optimization problem that has a non-convex and combinatorial search space so that several solution algorithms may converge to local optima. Therefore, many works have been proposed to solve the TEP problem considering its relaxation or reducing its search space. In any case, relaxation and reduction approaches should not compromise the quality of the final solution. This paper aims at analyzing the performance of a search space technique using a Constructive Heuristic Algorithm (CHA) admitting that the TEP problem is then solved using a Discreet Evolutionary Particle Swarm Optimization (DEPSO). On one hand the reduction quality is performed by analyzing whether the optimal expansion routes are included in the CHA constrained set and, on the other hand, the relaxation quality of the DC model is analyzed by checking if the optimal solution obtained with it violates any constraint using the AC model. The simulations were performed using three different test systems. The results suggest that the proposed CHA provides very good results in reducing the TEP search space and that the adoption of the DC model originates several violations if the full $\mathrm{AC}$ model is used to model the operation of the power system.
\end{abstract}

Index Terms -Multi-Year Transmission Expansion Planning, Constructive Heuristic Algorithm, Discrete Evolutionary Particle Swarm Optimization.

\section{INTRODUCTION}

The increasing demand for electricity requires planning the expansion of power systems in a careful way so that they can meet the future demand in a secure and reliable way. The changes on an existing power system usually correspond to build new generating facilities if possible close to demand centers or connecting sub-regions through transmission lines. However, the first option is frequently not economically feasible or even possible. Apart from that, the connection of sub-systems can enable the optimal dispatch of power plants. Therefore, the TEP problem aims at defining when, where and what equipment (transmission lines, cables or transformers) should be built in order to optimize a predetermined objective (minimizing or maximizing some function) along an extended horizon and meeting a number of constraints. The objectives to be optimized are usually:
- Minimization of the investment and operation costs;

- Increase of the system reliability;

- Minimization of the greenhouse gas emissions;

- Obtaining an improved voltage profile.

The TEP exercise can be conducted considering static or dynamic (multiyear) approaches. In the static TEP models the equipment selected to include in the network in one period are considered in the basis topology for the subsequent one which means that using a static approach for an extended horizon implies solving the TEP problem as many times as the time steps in that horizon. In multiyear approaches the problem is solved in a single run considering all periods in the same formulation. This means taking the horizon in a holistic way and thus turning it possible to obtain better quality long-term expansion plans.

In this paper a TEP multiyear approach was developed in order to minimize the system total cost. This cost reflects the investment and the operational cost, namely associated with Power Not Supplied (PNS), which is related to a penalty term in the objective function of the problem. The developed approach was applied to 3 different test systems considering that generation can be rescheduled. The main objectives of this paper are highlighted below:

- Evaluate the quality of the solution if a reduction search space technique using a CHA algorithm is used together with DC and AC Optimal Power Flow (OPF) models. This means checking whether the CHA is able to identify the main expansion routes without eliminating routes that are part of the final solution;

- Evaluate the quality of the expansion obtained using the DC-OPF model. This means checking if the expansion plan violates any operation constraint modeled using the AC power flow model.

Regarding the structure of the paper, after this Introduction, Section II presents the TEP mathematical model, Sections III and IV provide a brief description of the CHA and DEPSO 
techniques, Section $\mathrm{V}$ presents the simulation results and Section VI outlines the main conclusions of this work.

\section{TEP MATHEMATICAL FORMULATIONS}

\section{A. General TEP formulation}

The AC-OPF is the most adequate model to deal with TEP problems. However, the TEP problem is a challenging Mixed Integer Non-Convex Non-Linear Program (MINLP) and so several approaches can be used to turn it more tractable as the use of a DC based dispatch model instead of a full AC dispatch model. Following [1], the general formulation of the TEP problem is given by $(1-4)$.

Minimize $Z=\sum_{p=1}^{n p} \frac{C_{p}^{i n v}+\beta \cdot P N S_{p}}{(1+r)^{p}}$

Subject to:

Physical constraints;

Financial constraints;

Quality of service constraints.

Physical constraints are associated to the generator and branch capacity limits, financial constraints refer to the maximum amount that is available to be invested in a certain period and the quality of service constraints are for instance related with the maximum value allowed for PNS in normal or contingency regimes.

The objective function (1) is calculated through the $n p$ periods using the values of the investment $\operatorname{cost} C_{p}^{i n v}$ and the $P N S_{p}$ penalty cost for each period $p$ brought to the departing period using a return rate $r$. In each period, the value of PNS is obtained from an optimal dispatch problem using the $\mathrm{AC}$ or DC models as detailed in the next Section.

\section{B. AC Model based dispatch model}

The AC-OPF is formulated by (5) to (13).

$$
\begin{gathered}
\text { Min } C_{O P}=\sum_{g=1}^{n g} C_{g}(P) \\
\text { subject to } \quad(V, \theta, n)-P_{G}+P_{D}=0 \\
Q(V, \theta, n)-Q_{G}+Q_{D}=0 \\
P_{G \min } \leq P_{G} \leq P_{G \max } \\
Q_{G \text { min }} \leq Q_{G} \leq Q_{G \max } \\
V_{\text {min }} \leq V \leq V_{\max } \\
(N+\stackrel{o}{N}) S^{\text {from }} \leq(N+\stackrel{o}{N}) S_{\max } \\
(N+\stackrel{o}{N}) S^{t o} \leq(N+\stackrel{o}{N}) S_{\max } \\
0 \leq n \leq n_{\max }
\end{gathered}
$$

In this formulation $P(V, \theta, n)$ and $Q(V, \theta, n)$ are calculated by (14) and (15), and the bus conductance $G$ and susceptance B are given by (16) and (17).

$$
\begin{aligned}
& P(V, \theta, n)=V_{i} \sum V_{j}\left[G_{i j}(n) \cdot \cos \theta_{i j}+B_{i j}(n) \cdot \sin \theta_{i j}\right] \\
& Q(V, \theta, n)=V_{i} \sum V_{j}\left[G_{i j}(n) \cdot \sin \theta_{i j}-B_{i j}(n) \cdot \cos \theta_{i j}\right]
\end{aligned}
$$

$$
\begin{gathered}
G=\left\{\begin{array}{l}
G_{i j}(n)=-\left(n_{i j} \cdot g_{i j}+n_{i j} \cdot g_{i j}\right) \\
G_{i i}(n)=\sum_{j \in \Omega_{i}}\left(n_{i j} \cdot g_{i j}+n_{i j} \cdot g_{i j}\right)
\end{array}\right\} \\
B=\left\{\begin{array}{l}
B_{i j}(n)=-\left(n_{i j} \cdot b_{i j}+n_{i j}^{o} \cdot b_{i j}\right) \\
B_{i i}(n)=b_{i j}^{s h}+\sum_{j \in \Omega_{i}}\left[n_{i j}\left(b_{i j}+b_{i j}^{s h}\right)+n_{i j}^{o}\left(b_{i j}^{o}+b_{i j}^{o b}\right)\right]
\end{array}\right\}
\end{gathered}
$$

The apparent flows $S_{i j}{ }^{\text {from }}$ and $S_{i j}{ }^{t o}$ in branch ij are calculated by (18) and (19) where $P_{i j}^{\text {from }}, Q_{i j}^{\text {from }}, P_{i j}^{t o}$ and $Q_{i j}^{t o}$ are given by (20) to (23).

$$
\begin{gathered}
S_{i j}^{\text {from }}=\sqrt{\left(P_{i j}^{\text {from }}\right)^{2}+\left(Q_{i j}^{\text {from }}\right)^{2}} \\
S_{i j}^{t o}=\sqrt{\left(P_{i j}^{t o}\right)^{2}+\left(Q_{i j}^{t o}\right)^{2}} \\
P_{i j}^{\text {from }}=V_{i}^{2} \cdot g_{i j}-V_{i} \cdot V_{j}\left(g_{i j} \cdot \cos \theta_{i j}+b_{i j} \cdot \sin \theta_{i j}\right) \\
Q_{i j}^{\text {from }}=-V_{i}^{2} \cdot\left(b_{i j}{ }^{s h}+b_{i j}\right)-V_{i} \cdot V_{j}\left(g_{i j} \cdot \sin \theta_{i j}-b_{i j} \cdot \cos \theta_{i j}\right) \\
P_{i j}^{t o}=V_{j}^{2} \cdot g_{i j}-V_{i} \cdot V_{j}\left(g_{i j} \cdot \cos \theta_{i j}-b_{i j} \cdot \sin \theta_{i j}\right) \\
Q_{i j}^{t o}=-V_{j}^{2} \cdot\left(b_{i j}{ }^{s h}+b_{i j}\right)+V_{i} \cdot V_{j}\left(g_{i j} \cdot \sin \theta_{i j}+b_{i j} \cdot \cos \theta_{i j}\right)
\end{gathered}
$$

In this formulation, the objective function (5) corresponds to the system operation cost with $n g$ generation units, which in turn is the sum of the dispatch in each unit $g . P_{G}$ and $Q_{G}$ are the real and reactive power generation, $P_{D}$ and $Q_{D}$ are the real and reactive power demand, $V$ is the voltage magnitude, $S_{i j}{ }^{\text {from }}$ and $S_{i j}{ }^{\text {to }}$ are the branch apparent flows in both terminals, and $g_{i j}$ and $b_{i j}$ are the conductance and the susceptance of branch i-j, $\stackrel{o}{N}$ and $N$ are diagonal matrices containing the equipment in the base topology and the added equipment respectively.

\section{DC Model}

The DC OPF model is the most popular approximation to the AC OPF model. According to [2], this model admits that the voltage magnitudes are close to $1 \mathrm{pu}$, branch susceptance are much larger than the corresponding branch conductance and the phase angle difference across each branch is negligible. As a result $\sin \left(\theta_{i}-\theta_{j}\right) \approx \theta_{i}-\theta_{j}$ and $\cos \left(\theta_{i}-\theta_{j}\right) \approx 1$ and so equation (20) is approximated by (24).

$$
P_{i j}=b_{i j} \cdot\left(\theta_{i}-\theta_{j}\right)
$$

\section{Constructive Heuristic Algorithm to ReducE THE SEARCH SPACE}

CHAs are tools that use a sensitivity function to iteratively drive the selection of new elements to include in the solution till the final plan is obtained. In general, the output of CHA algorithms include a number of branches larger than the ones in an optimal plan which means that their output can be used as input in a subsequent optimization procedure to obtain the final plan. If CHA's are used like this, that is to reduce the search 
space, the performance of the subsequent optimization module can be largely improved. More information about the CHA procedures can be accessed in [3]. The following pseudo code briefly details the main steps of the Least Effort CHA.

\section{Procedure CHA}

Do

Check if the base topology presents PNS for a determined horizon plan.

Repeat

Solve the Optimal Power Flow model

Check the most congested equipment in the system

Insert an equipment in parallel to this one

Update the system with the new equipment

Until the current topology does not present PNS

\section{End Least Effort CHA}

\section{Discrete EVOLUTIONARY PARTICLE SWARM OPTIMIZATION}

DEPSO was originally reported in [4] and it provides an adaptation of the Evolutionary Particle Swarm Optimization to solve problems with non-continuous and integer search spaces as the TEP problem. This tool combines the main features of evolutionary computing and swarm intelligence using the main blocks of Genetic Algorithm and Particle Swarm Optimization. Typically, the planner provides a list of $N_{\text {proj }}$ equipment that can be used for network expansion. Each particle in DEPSO represents a possible expansion plan and it is encoded by a vector that has $N_{\text {proj }}$ positions. Each position is filled with integer values ranging from 0 to $N p$ (number of periods considered in the study) indicating in which period it is included in the network. If 0 , then the corresponding equipment is not used in this expansion plan.

The most remarkable features of this tool is the computational effort required to find optimal and sub-optimal solutions. This characteristic is derived from the evolutionary part of the algorithm. On the other hand, the quality of the final solution is closely related to the initial swarm, i.e., the final expansion plan will be better if the particles start their journey from good positions in the search space. This feature is enhanced because DEPSO is a multi-agent population algorithm. These features are very adequate to solve the TEP problem namely if a CHA is used as an initial step because:

- reduction of the search space conducted by the CHA This can significantly reduce the computational effort and at the same time provides a good initialization for the particles in departing population thereby improving the performance of DEPSO;

- Evaluate the quality of the solution if a DC based dispatch is used - the use of the DC model can also significantly reduce the computational effort associated with the DEPSO.

Therefore, these two features are in line with the main objective of this paper, which is to analyze if these approaches (reduction and approximation) imply any kind of compromise on the goodness of the final expansion plan.

The main DEPSO blocks are presented below.

\section{Procedure DEPSO}

Do

Set the list of candidate equipment having $N_{p r o j}$ elements Initialize a random population with $p s$ particles

Repeat

\section{Replication \\ Mutation}

Recombination

Evaluation

Selection

Stop Test

Until test is positive

\section{End DEPSO}

In the replication block, the swarm is replicated $r$ times and the elements of these $r$ clones are then mutated in order to increase the diversity of the search. In the mutation block the weights and the best particle obtained until now are mutated. Then, in the recombination block the particles in the swarm share the information to move through the search space. This recombination step uses the usual recombination rule of PSO algorithms according to which a particle move is determined by an inertial, a memory and a cooperation term. The inertia term tends to move the particle in the same direction as in the previous iteration and the memory term models the individual knowledge of the particle because the move is attracted to the position of the best of its ancestors. The cooperation term represents the collective knowledge and it attracts the particle to the best global position obtained so far. The combination of these three terms determines the position of the new particle in the next iteration. All the particles are then evaluated using an OPF model (DC or AC) in each of the periods in the horizon and checking the values of PNS. Non zero PNS values penalize the fitness function of each particle. The values of the fitness function of each particle in each clone are then used in the selection block to run an elitist selection. Detailed information on the DEPSO algorithm is available in [3].

\section{TESTS AND RESUltS}

The CHA and the DEPSO tools were applied to 3 different test systems indicated below. Case 6 and Case 30 Bus test systems are available in version 5.1 of the MATPOWER tool described in [5] and the third system is a modified version of the IEEE RTS 24 bus system. The objective was to analyze if the CHA reduces the search space not eliminating routes that are used in the optimal final expansion plan and to analyze if the solutions using the DC based dispatch model do not violate constraints of the AC model. The simulation were performed in Matlab ${ }^{\circledR}$ with an Intel i7, 3.4GHz, 8 GB RAM.

Regarding the power flow solution, the maximum allowed flow was considered in emergency condition for all branches and the loads are modeled as dispatchable loads, that is, as 
negative real power injections with associated negative costs as detailed in [6].

The tests were performed using the multiyear TEP model with 3 periods and admitting a 5\% load increase per period. DEPSO was run using 150 particles in the swarm, the PNS penalty factor used in (1) was set at $\beta=10^{12}$. The DEPSO algorithm stops when the best particle does not change after running 50 iterations. Finally, the $\mathrm{P}$ communication factor that controls the cooperation term in the DEPSO recombination rule was set at 1 as in classical applications.

\section{A. Case 6 Bus Test System}

The investment cost for each branch was taken as proportional to capacity of that branch and the generation capacity and loads are tripled to turn the grid more stressed. The results of the simulations are described below.

- $\quad$ Reduction of the search space

The CHA was used in the Case 6 bus test system and was applied in AC-OPF and DC-OPF. In both cases the process took less than 1 minute and the results are shown in Table I.

TABLE I. REDUCTION IN THE CASE 6 BUS TEST SYSTEM

\begin{tabular}{|c|c|}
\hline MODEL & EQUIPMENT \\
\hline AC OPF & $1-4,1-5,2-4,3-6$ \\
\hline DC OPF & $2-4,2-5,3-5,3-6$ \\
\hline
\end{tabular}

- $\quad$ Solutions obtained using the entire search space

DEPSO was applied to the Case 6 bus using the AC and DC approaches. In the AC-OPF model the process solved 283053 OPFs and in DC version solved model 198453 OPFs. The results obtained are shown in Table II.

TABLE II. DEPSO APPLIED TO THE CASE 6 BUS - ENTIRE SEARCH SPACE

\begin{tabular}{|c|c|c|c|}
\hline MODEL & PERIOD & EQUIPMENT & $\begin{array}{c}\text { INV. COST } \\
(\boldsymbol{€})\end{array}$ \\
\hline \multirow{3}{*}{ AC } & 1 & $\begin{array}{c}1-4,(2) 1-5,(3) 2-4,(2) 2-5,(2) \\
2-6,3-5,(2) 3-6\end{array}$ & $790.10^{6}$ \\
\cline { 2 - 4 } OPF & 2 & $3-5$ & $70.10^{6}$ \\
\cline { 2 - 4 } & 3 & $1-2,1-4,(2) 2-3$ & $180.10^{6}$ \\
\hline \multirow{2}{*}{ DC } & 1 & $(3) 1-5,(2) 2-4,3-6$ & $390.10^{6}$ \\
\cline { 2 - 4 } OPF & 2 & $3-5$ & $70.10^{6}$ \\
\cline { 2 - 4 } & 3 & $1-2,1-4,4-5,(2) 5-6$ & $200.10^{6}$ \\
\hline
\end{tabular}

- $\quad$ Solutions obtained with the reduced search space

The DEPSO was also applied to Case 6 bus using the AC and the DC-OPF using the reduced search space. In the first case the process solves $110253 \mathrm{AC}-\mathrm{OPFs}$ and in the second case 92253 DC-OPFs. The results are shown in Table III.

TABLE III. DEPSO APPLIED TO THE CASE 6 BUS - REDUCED SEARCH SPACE

\begin{tabular}{|c|c|c|c|}
\hline \multirow{2}{*}{ MODEL } & PERIOD & EQUIPMENT & $\begin{array}{c}\text { INV. } \\
\text { COST (€) }\end{array}$ \\
\hline \multirow{2}{*}{ AC } & 1 & $(3) 1-4,(3) 1-5,(3) 2-4,(3) 3-6$ & $720.10^{6}$ \\
\cline { 2 - 4 } OPF & 2 & --- & 0 \\
\cline { 2 - 4 } & 3 & --- & 0 \\
\hline \multirow{2}{*}{ DC } & 1 & $(2) 2-4,3-5,(2) 3-6$ & $350.10^{6}$ \\
\cline { 2 - 4 } OPF & 2 & --- & 0 \\
\cline { 2 - 4 } & 3 & $2-4,(2) 2-5,3-5$ & $540.10^{6}$ \\
\hline
\end{tabular}

\section{B. Modified IEEE RTS 24 Bus}

In this case we used the modified IEEE 24 Bus RTS system that has some differences regarding the original one proposed in [8]. The details of this modified system can be found in [3] and [6]. The results of the simulations are described as follows.

- $\quad$ Reduction of the search space

The CHA was used in the modified 24 Bus RTS test system using the AC-OPF and the DC-OPF. In both cases the process took less than 1 minute, the obtained results are shown in Table IV.

TABLE IV. REDUCTION IN THE MODIFIED 24 BUS SYSTEM

\begin{tabular}{|c|c|}
\hline MODEL & EQUIPMENT \\
\hline $\mathrm{AC}$ & $1-5,3-24,6-10,7-8,11-13,13-23$, \\
$\mathrm{OPF}$ & $14-16,15-24,16-17,17-18$ \\
\hline $\mathrm{DC}$ & $3-24,6-10,7-8,11-13,14-16,16-17$ \\
$\mathrm{OPF}$ & \\
\hline
\end{tabular}

- $\quad$ Solutions obtained Using the entire search space

DEPSO was applied to the modified 24 bus using the $\mathrm{AC}$ and the DC approaches. In the AC-OPF model the process solved 123906 OPFs and in DC-OPF model 277653 OPFs. The results are shown in Table $\mathrm{V}$.

TABLE V. DEPSO APPLIED IN THE MODIFIED 24 BUS SYSTEM - ENTIRE SEARCH SPACE

\begin{tabular}{|c|c|c|c|}
\hline MODEL & PERIOD & EQUIPMENT & $\begin{array}{c}\text { INV. COST } \\
(\boldsymbol{\epsilon})\end{array}$ \\
\hline \multirow{2}{*}{$\mathrm{AC}$} & 1 & $6-10,7-8,14-16$ & $86.10^{6}$ \\
\cline { 2 - 4 } OPF & 2 & --- & 0 \\
\cline { 2 - 4 } & 3 & $9-11,10-12,15-21$ & $236.10^{6}$ \\
\hline \multirow{2}{*}{$\mathrm{DC}$} & 1 & $6-10,14-16$ & $70.10^{6}$ \\
\cline { 2 - 4 } OPF & 2 & --- & 0 \\
\cline { 2 - 4 } & 3 & $1-3,(2) 15-21,19-20,21-22$ & $531.10^{6}$ \\
\hline
\end{tabular}

- $\quad$ Solutions founded on the reduced search space

The DEPSO was also applied in modified 24 bus using AC and DC-OPF using the entire search space solving 101103 and 142653 OPFs respectively. The results obtained are shown in table VI below:

TABLE VI. DEPSO APPLIED TO THE MODIFIED 24 BUS SYSTEM REDUCED SEARCH SPACE

\begin{tabular}{|c|c|c|c|}
\hline MODEL & PERIOD & EQUIPMENT & $\begin{array}{c}\text { INV. COST } \\
(\boldsymbol{(})\end{array}$ \\
\hline \multirow{2}{*}{$\mathrm{AC}$} & 1 & $6-10,7-8,14-16$ & $86.10^{6}$ \\
\cline { 2 - 4 } OPF & 2 & --- & 0 \\
\cline { 2 - 4 } & 3 & $6-10,16-17,17-18$ & $72.10^{6}$ \\
\hline \multirow{2}{*}{ DC } & 1 & $6-10,14-16$ & $70.10^{6}$ \\
\cline { 2 - 4 } OPF & 2 & --- & 0 \\
\cline { 2 - 4 } & 3 & $16-17$ & $36.10^{6}$ \\
\hline
\end{tabular}

\section{Case 30 Bus Test System}

This system was obtained from MATPOWER version 5.1 and its generation capacities and loads were also tripled.

\section{- $\quad$ Reduction of the search space}

The CHA was used in the Case 30 Bus test system applying the $\mathrm{AC}-\mathrm{OPF}$ and the DC-OPF. In both cases the process took 
less than 1 minute and the obtained results are shown in Table VII.

TABLE VII. REDUCTION IN THE CASE OF THE 30 BUS TEST SYSTEM

\begin{tabular}{|c|c|}
\hline MODEL & EQUIPMENT \\
\hline AC OPF & $2-6,6-8,21-22,22-24,27-30$ \\
\hline \multirow{2}{*}{ DC OPF } & $2-6,6-8,12-13,15-18,10-20,21-22,23-24,25-27,27-$ \\
& 30 \\
\hline
\end{tabular}

- $\quad$ Solutions obtained using the entire search space

DEPSO was applied in the Case 30 bus using the AC and the DC approaches. In this case 389253 and 245253 OPFs are solved respectively. The results are shown in Table VIII.

TABLE VIII. DEPSO APPLIED IN THE CASE 30 BUS - ENTIRE SEARCH SPACE

\begin{tabular}{|c|c|c|c|}
\hline MODEL & PERIOD & EQUIPMENT & $\begin{array}{c}\text { INV. COST } \\
(\boldsymbol{\epsilon})\end{array}$ \\
\hline \multirow{4}{*}{ AC } & 1 & $\begin{array}{r}2-6,(3) 6-8,10-20,10-22,15- \\
23,(2) 21-22,27-29\end{array}$ & $353.10^{6}$ \\
\cline { 2 - 4 } OPF & 2 & $\begin{array}{r}4-12,6-10,6-28,27-28, \\
(2) 29-30\end{array}$ & $210.10^{6}$ \\
\cline { 2 - 4 } & 3 & $\begin{array}{c}(2) 1-2,2-5,3-4,4-6,4-12,6-7, \\
6-9,8-28,9-10,10-21,10-22, \\
(2) 12-14,12-15,(2) 12-16, \\
15-18,19-20,25-26\end{array}$ & $1255.10^{6}$ \\
\hline \multirow{3}{*}{ DC } & 1 & $2-6,(2) 6-8,21-22,27-30$ & $177.10^{6}$ \\
\cline { 2 - 4 } OPF & 2 & $15-18,15-23$ & $32.10^{6}$ \\
\cline { 2 - 4 } & 3 & $\begin{array}{r}1-2,(2) 3-4,5-7,8-28,12-16, \\
29-30\end{array}$ & $540.10^{6}$ \\
\hline
\end{tabular}

- $\quad$ Solutions obtained using the reduced search space

The DEPSO was also applied in Case 30 Bus test system using the AC and the DC-OPF on the reduced search space. In this case 115653 and 117453 OPFs are solved respectively. The results obtained are shown in table IX below.

TABLE IX. DEPSO APPLIED IN THE CASE 30 BUS - REDUCED SEARCH SPACE

\begin{tabular}{|c|c|c|c|}
\hline MODEL & PERIOD & EQUIPMENT & $\begin{array}{c}\text { INV. CosT } \\
(€)\end{array}$ \\
\hline \multirow{3}{*}{$\begin{array}{l}\mathrm{AC} \\
\mathrm{OPF}\end{array}$} & 1 & $\begin{array}{c}\text { (3) } 2-6, \text {, (3) } 6-8, \text {, (2) } 21-22 \\
\text { (3) } 22-24,27-30\end{array}$ & $419.10^{6}$ \\
\hline & 2 & -- & 0 \\
\hline & 3 & $21-22,(2) 27-30$ & $64.10^{6}$ \\
\hline \multirow{3}{*}{$\begin{array}{l}\mathrm{DC} \\
\mathrm{OPF}\end{array}$} & 1 & $2-6,(2) 6-8,21-22,27-30$ & $177.10^{6}$ \\
\hline & 2 & $10-20$ & $32.10^{6}$ \\
\hline & 3 & $2-6,(2) 12-13,25-27,27-30$ & $227.10^{6}$ \\
\hline
\end{tabular}

TABLE X. QUALITY SOLUTION ANALYSIS - DC-OPF MODEL

\begin{tabular}{|c|c|c|}
\hline \multirow{2}{*}{ TEST SYSTEM } & $\begin{array}{c}\text { SOLUTION FOUNDED USING } \\
\text { DC-OPF }\end{array}$ & $\begin{array}{c}\text { PNS IN AC-OPF } \\
\text { (MW) }\end{array}$ \\
\hline \multirow{2}{*}{ Case 6 bus } & REDUCED SEARCH SPACE & 533,07 \\
\cline { 2 - 3 } & ENTIRE SEARCH SPACE & 462,10 \\
\hline \multirow{2}{*}{ RTS 24 bus } & REDUCED SEARCH SPACE & 465,64 \\
\cline { 2 - 3 } Case 30 bus & ENTIRE SEARCH SPACE & 448,51 \\
\cline { 2 - 3 } & REDUCED SEARCH SPACE & 199,05 \\
\hline
\end{tabular}

\section{Evaluation of the quality of the DC solution}

In order to evaluate the solution quality of the DC model, the DC solutions are checked using the AC-OPF. The results obtained are shown in Table X.

\section{CONCLUSIONS}

In this paper a multi-year Transmission Expansion Planning was conducted using AC and DC optimal power flow models. Besides, the Constructive Heuristic Algorithm presented in Section III was applied to reduce the search space using the AC and DC model. The simulations were performed in three different test systems termed as Case 6 bus and Case 30 bus from version 5.1 of the MATPOWER tool and the modified IEEE RTS 24 Bus from [3].

Regarding the search space reduction exercise, from the results presented in the above section, the AC model shows excellent performance in all three test systems since it was able to ensure that the best branches were included in the reduced search space, that is, the branches that belong to the best expansion plan were maintained in the search space after the reduction. On the other hand, the $\mathrm{CHA}$ using the DC model does not present this type of performance.

The results obtained using the DC model were then input on the $\mathrm{AC}$ to check if there were violations of $\mathrm{AC}$ constraints. In all cases, the best DC solution has non zero PNS when using the AC model. These results are in line with the results reported in [2], that is, TEP approaches using the DC models are likely to present violations using the $\mathrm{AC}$ model.

\section{ACKNOWLEDGMENT}

P. Vilaça acknowledges the financial support from CAPES Scholarship under Bex 13207-13-0.

\section{REFERENCES}

[1] M. C. Da Rocha and J. T. Saraiva, "A multiyear dynamic transmission expansion planning model using a discrete based EPSO approach," Electr. Power Syst. Res., vol. 93, pp. 83-92, 2012.

[2] R. Bent, C. Coffrin, R. R. E. Gumucio, and P. Van Hentenryck, "Transmission Network Expansion Planning: Bridging the gap between AC heuristics and DC approximations," in 2014 Power Systems Computation Conference, 2014, pp. 1-8.

[3] P. V. Gomes and J. T. Saraiva, "Hybrid Discrete Evolutionary PSO for AC Dynamic Transmission Expansion Planning," in IEEE Energycon conference, 2016.

[4] M. J. C. da Rocha and J. T. Saraiva, "Discrete Evolutionary Particle Swarm Optimization for Multiyear Transmission Expansion Planning," in Power Systems Computation Conference, 2011.

[5] R. D. Zimmerman, C. E. Murillo-Sanchez, and R. J. Thomas, "MATPOWER: Steady-State Operations, Planning, and Analysis Tools for Power Systems Research and Education," IEEE Trans. Power Syst., vol. 26, no. 1, pp. 12-19, Feb. 2011.

[6] P. V. Gomes and J. T. Saraiva, "Hybrid Genetic Algorithm for MultiObjective Transmission Expansion Planning," in IEEE Energycon conference, 2016.

[7] M. J. Rider, A. V. Garcia, and R. Romero, "Power system transmission network expansion planning using AC model," IET Gener. Transm. Distrib., vol. 1, no. 5, p. 731, Sep. 2007.

[8] P. Subcommittee, "IEEE Reliability Test System," IEEE Trans. Power Appar. Syst., vol. PAS-98, no. 6, pp. 2047-2054, Nov. 1979.

[9] S. Haffner, A. Monticelli, A. Garcia, J. Mantovani, and R. Romero, "Branch and bound algorithm for transmission system expansion planning using a transportation model," IEE Proc. - Gener. Transm. Distrib., vol. 147, no. 3, p. 149, May 2000. 